\title{
Treatment of Large Osteochondral Defects with Spongiosaplasty and Collagen Type I Hydrogel-Based Autologous Chondrocyte Implantation
}

\author{
Johannes C. Reichert ${ }^{1 *}$, Florian Fensky², Sebastian Siebenlist ${ }^{3}$, Ulrich Noth ${ }^{1}$, Lars Rackwitz ${ }^{1}$ \\ ${ }^{1}$ Department of Orthopaedic and Trauma Surgery, Ev. Waldkrankenhaus Spandau, Stadtrandstr. 555, 13589, Berlin, Germany \\ ${ }^{2}$ Department of Trauma, Hand- and Reconstructive Surgery, University Medical Center Hamburg-Eppendorf, Martinistraße 52, 20246 \\ Hamburg, Germany \\ ${ }^{3}$ Department of Trauma Surgery, Klinikum rechts der Isar, Technical University Munich, Ismaninger Road 22, 81675 Munich, Germany
}

Received: February 01, 2017; Accepted: March 19, 2017; Published: May 19, 2017

*Corresponding author: Johannes C. Reichert, MD, PhD, Department of Orthopaedic and Trauma Surgery, Ev. Waldkrankenhaus Spandau, Stadtrandstr. 555, 13589 Berlin, Germany, Tel:+49-30-3702-1065;E-mail: johannes.reichert@pgdiakonie.de

\section{Abstract}

Clinical data suggest that the reconstruction of large osteochondral defects requires application of osseous cylinders to augment the bony defect. This prospective case series investigates the clinical outcome after impaction grafting as an alternative to osseous cylinder transplantation to reconstruct the subchondral bone plate.

We report on three patients with large osteochondral defects of the femoral condyle (averaging $10.9 \mathrm{~cm}^{2}$ ) resulting from osteochondritis dissecans or osteonecrosis. Defects were treated with spongiosaplasty followed by matrix-based autologous chondrocyte implantation (MACI) using a collagen-I-hydrogel. The patients were examined at 3, 6, 12, 24, 36, 48, and 60 months after surgery. The follow-up included radiographs, MRI, patient/doctor assessment as well as the IKDC-2000 evaluation package (IKDC score).

At final follow-up, all three patients were satisfied with the long-term outcome since free of pain. Their functional and activity levels were back to normal, which was also reflected in the obtained IKDC-scores. Radiographic and MRI imaging showed complete reconsolidation of the subchondral osseus defects and excellent integration of the implanted collagen based matrix with the surrounding hyaline cartilage.

Therefore, autologous spongiosaplasty as an alternative to the transplantation of osseous cylinders is a sufficient technique to reconstruct the subchondral bone plate in patients with large osteochondral defects.

KeyWords: osteochondral defect; femoral condyle; spongiosaplasty; autologous chondrocyte implantation (ACI); collagen hydrogel

\section{Introduction}

Large chondral lesions are often an expression of damage at the tidemark-subchondral bone interface. As a result, treatment strategies should aim to restore a functional cartilagebone unit. Most techniques established to treat chondral defects have therefore proven to be insufficient for the treatment of lesions exceeding a size of $4 \mathrm{~cm}^{2}$ (1-4). Cartilage lesions of larger dimensions are commonly treated by osteochondral grafting $\left(\mathrm{OATS}^{\mathrm{TM}}, \mathrm{OCT}\right) /$ mosaicplasty or autologous chondrocyte implantation $(\mathrm{ACI})(5,6)$. However, donor sites for OCT are limited, the harvesting process itself may cause symptoms and disability, and the impaction during OCT can lead to chondrocyte apoptosis and compromise proteoglycan turnover resulting in graft failure while the cartilage graft will not inherently bond to a recipient site. As a results cartilage lesions of larger dimensions $\left(>4 \mathrm{~cm}^{2}\right)$ are commonly treated by ACI. Notably, cartilage repair tissue following ACI morphologically is of hyaline cartilage like appearance. Biomechanically, it can reach up to $90 \%$ stiffness of genuine hyaline cartilage providing long-term graft integrity even in larger sized defects (7). Recently, the concept of matrix assisted autologous chondrocyte implantation (MACI) has advanced as a further promising treatment option (8). Essentially, the concept is based on the use of biodegradable polymers as temporary scaffolds for the in vitro growth of living cells and their subsequent transplantation into the defect site. The use of threedimensional scaffolds has been shown to favor the maintenance of the differentiated chondrocyte phenotype (9). But to restore the integrity of subchondral bone and to provide a mechanically stable counter bearing seems to be crucial to achieve a viable good quality regenerate, congruity of the grafted surface, and sufficient transplant integration, especially in large osteochondral defects.

In the following we describe a method to provide a mechanically sufficient transplant bed prior to MACI using the CaReS® system (Arthro Kinetics plc, Krems, Austria) without the need to transplant osseous cylinders presenting three clinical cases with a 5-year follow-up. 


\section{Patients and methods}

The study was reviewed by the institutional ethics committee and therefore performed in accordance with the ethical standards stated in the declaration of Helsinki. All subjects included gave their informed consent prior to inclusion in the presented study.

\section{Patient one (P1)}

Thirty-year-old male software engineer diagnosed with a $4 \times 2.3 \mathrm{~cm}\left(9.2 \mathrm{~cm}^{2}\right)$ measuring OD and a posteriorly localized bone cyst of the lateral femoral condoyle (Fig. 1a). Previous surgery: Pridie drilling at the age of 20.
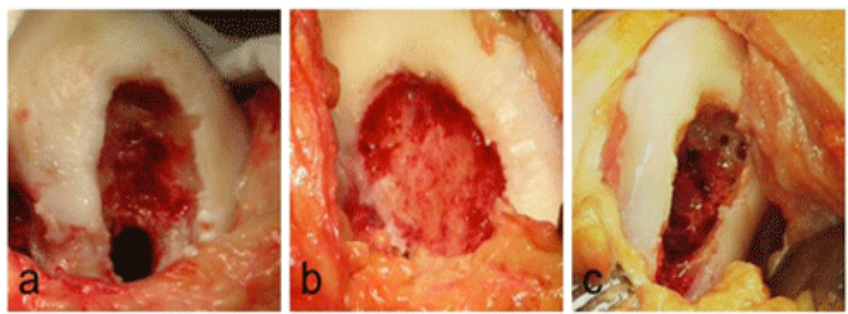

Figure 1: Osteochondral defects of the three patients before reconstruction. (a) Osteochondritis dissecans (OD) stage IV of the lateral femoral condyle with a bone cyste in the posterior part (P1); (b) OD stage IV of the medial femoral condyle (P2); (c) Steroid-induced osteonecrosis $(\mathrm{ON})$ of the lateral femoral condyle (P3).

\section{Patient two (P2)}

Thirty-one-year-old male steelworker diagnosed with a $3 \times 4.2 \mathrm{~cm}\left(12.6 \mathrm{~cm}^{2}\right)$ measuring OD of the medial femoral condoyle (Fig. 1b). No previous surgeries.

\section{Patient three (P3)}

Thirty-four-year-old female medical pedagogue diagnosed with a $5 \times 2.2 \mathrm{~cm}\left(11 \mathrm{~cm}^{2}\right)$ measuring, steroid induced ON of the lateral femoral condyle (Fig. 1c). Previous surgery: Pridie drilling at the age of 24 .

All defects were classified as a stage IV defect according to the classification of the International Cartilage Repair Society (ICRS)(10). Indication for surgery was based on the recommendations of the German working group "Tissue Regeneration and Tissue Substitutes" for ACI (11).

\section{Surgical technique and postoperative management}

Knee arthroscopy was performed to determine the defect size and indication for ACI. Two cartilage biopsies were taken from the femoral notch (supero-medially, $2 \mathrm{~mm}$ in diameter). Chondrocytes were isolated by collagenase digestion and cultivated in patient own serum for 14 days prior to embedding in collagen I hydrogels of $8 \mathrm{~mm}$ height and $34 \mathrm{~mm}$ in diameter (CaReS®, Arthro Kinetics plc, Krems, Austria).

In a second, open procedure, the defect was exposed by a mini arthrotomy (Fig. 2a) and sharply excised and curetted (Fig. 2b). The underlying sclerotic bone was partially removed with a ball burr. A $2.0 \mathrm{~mm} \mathrm{~K}$-wire was used to drill holes through the sclerotic bone into the healthy underlying bone. The subchondral bone defect was reconstructed by impacting cancellous bone pieces (spongiosaplasty) harvested from the proximal tibia extending the surgical approach (P1 and P2) or from the iliac crest (P3)(Fig. 2c). The impacted bone was sealed with fibrin glue (TissuCol ${ }^{\circledR}$, Baxter, Unterschleißheim, Germany) and the chondrocytes containing hydrogel was implanted into the defect to be flush with the surrounding native cartilage (Fig. 2d, e). The joint was brought to full extension and closed without insertion of an intraarticular drain.
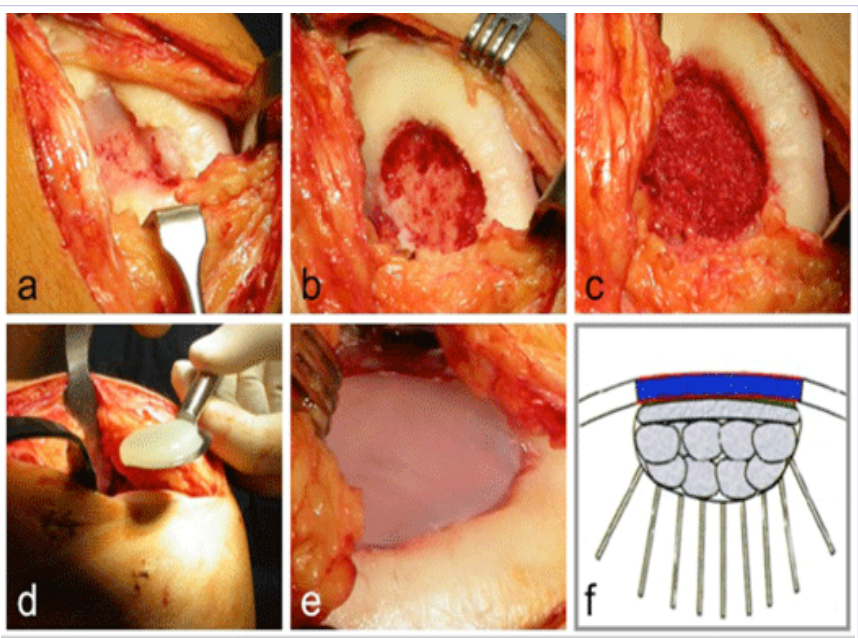

Figure 2: Surgical procedure illustrated for P2: OD of the medial femoral condyle before (a) and after debridement (b) and spot drilling the subchondral bone. (c) Reconstruction of the bone defect by impacting autologous bone. (d) Implantation of the collagen hydrogel to restore the cartilaginous joint surface (e). Schematic illustrating the surgical technique (f).

Postoperatively, the patients were immobilized in a brace $\left(10^{\circ}\right.$ of flexion) for 48 hours without exchanging the dressing to allow sufficient adhesion of the collagen gel to the bone graft. Continuous passive motion (CPM) commenced after 48 hours (10 hours a day) and continued until discharge. The range of motion was limited to $60^{\circ}$ of flexion for a period of 6 weeks without weight bearing. Thereafter, partial weight bearing (20kg) was allowed for 6 weeks, with unrestricted weight bearing afterwards. Physiotherapy to actively train muscle tone, power and proprioception was prescribed. Sports activities involving low compressive stress levels, such as cycling and swimming, were resumed after 6 months. Activities resulting in high axial stress peaks had to be avoided for 12 months postoperatively.

\section{Clinical and radiographic evaluation}

The IKDC-2000 evaluation package (10) was used to assess the clinical status prior to surgery as well as at the follow-up examinations at $3,6,12,24,36,48$, and 60 months after surgery. Also, patient and doctor satisfaction regarding the operative outcome was recorded using a global assessment scale 
(excellent, good, moderate, bad, very bad). Moreover, radiographs in the anterior-posterior and lateral plane as well as MRI scans were taken at 6,12, 24, 36 and 60 months after surgery. Mean and standard deviation (SD) were calculated using Microsoft Excel 2008.

\section{Results}

At the three month follow-up, two of the patients showed moderate swelling and intra-articular effusion, which had disappeared after 6 months. At the 24 month follow-up, all patients reported to be pain free under full weight bearing and had reached the sports activity level documented prior to surgery.

Global patient assessment increased from very bad (P1, P2 and P3) to good (P2) and excellent (P1 and P3), global doctor assessment increased from bad (P2) and very bad (P1 and P3) to excellent (P1, P2 and P3).

Radiographs showed good remodelling of the impacted bone. MRI images revealed good bonding of the transplanted hydrogel to the spongiosaplasty and to the healthy surrounding articular cartilage. Also, an excellent congruity of the hydrogel with the convexity of the condyle was achieved (Fig. 3).
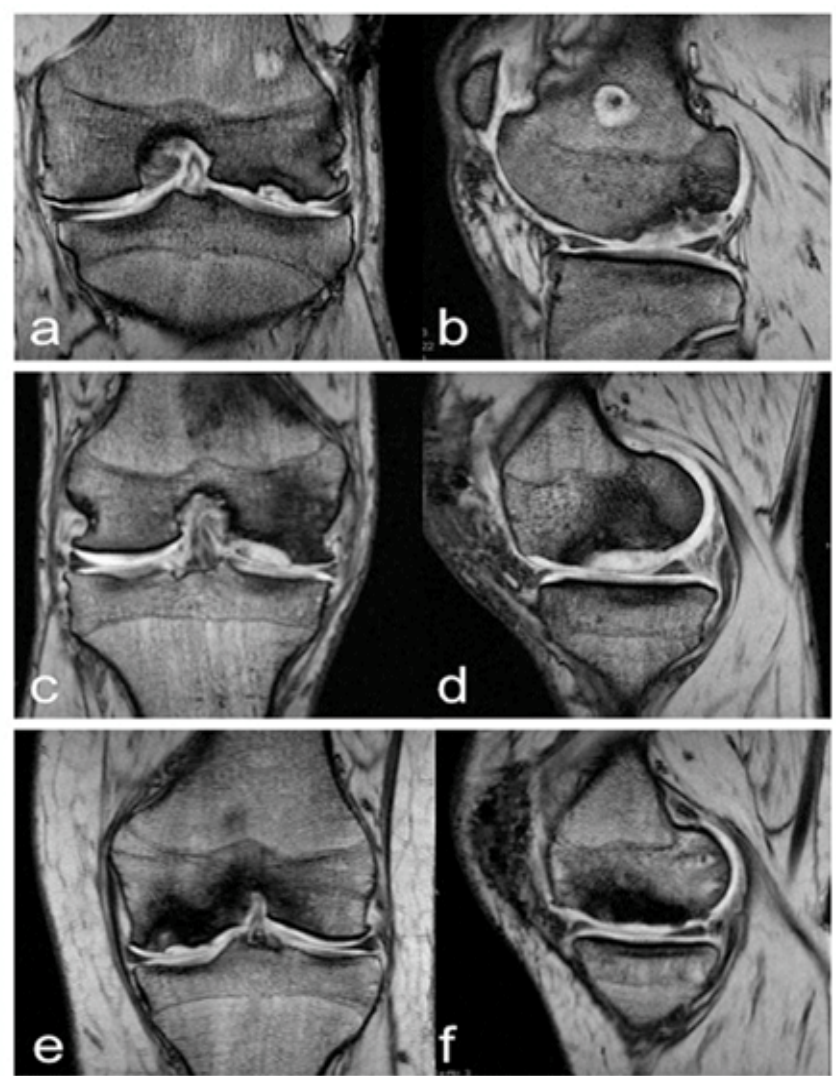

Figure 3: MRI findings at the final follow-up at 60 months (T1-weighted, frontal and sagittal plane) for P1 (a, b) P2 (c, d) and P3 (e, f). In all three patients a well integrated hydrogel reconstructing the articular surface was seen. The newly formed cartilage area showed no fissures at the surface.
The IKDC score increased from $47.50 \pm 13.7$ prior to surgery to $67.44 \pm 16.5$ at 3 months, $52.07 \pm 21.3$ at 6 months, $73.57 \pm 2.0$ at 12 months, $86.57 \pm 7.7$ at 24 months, $93.47 \pm 6.9$ at 36 months, and $83.90 \pm 7.0$ at 48 months (Fig. 4).

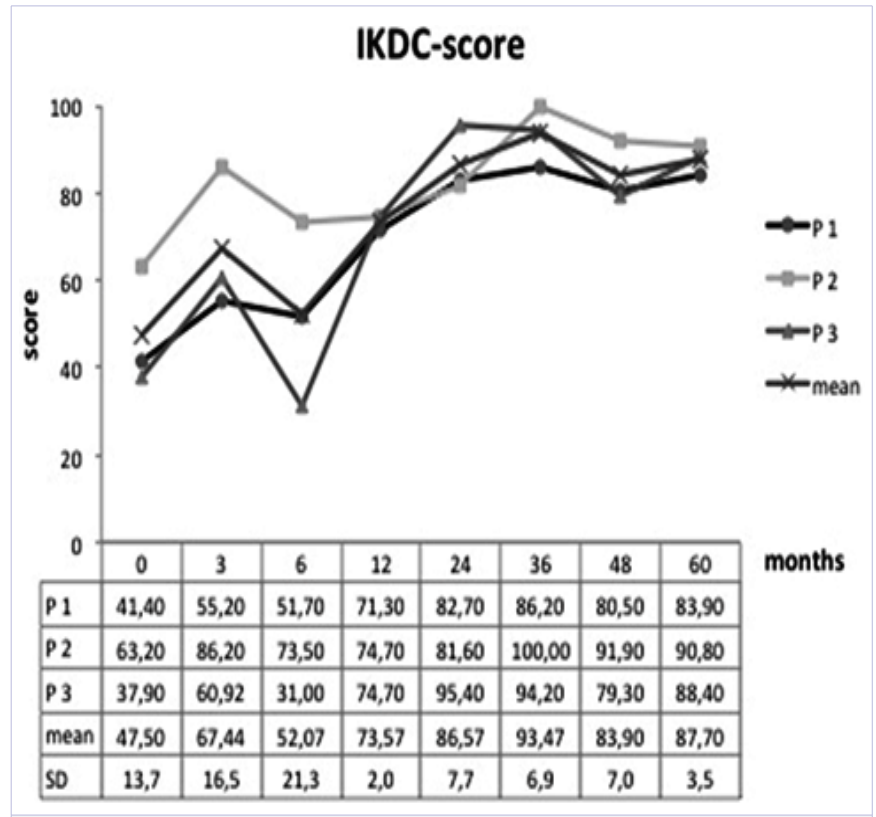

Figure 4: 1IKDC-scores of the three patients (P1-P3) after a follow-up period of 60 months. Also, the mean IKDC score \pm SD is given for the different follow-up time points.

In none of the patients peri- or postoperative complications were observed. No revision surgery had to be performed.

\section{Discussion}

For large osteochondral defects $\left(>9.6 \mathrm{~cm}^{2}\right)$ of weightbearing areas of the knee, techniques established for smaller sized lesions such as drilling, microfracturing, and transplantation of osteochondral plugs (OATS, Mega-OATS) provide only insufficient clinical outcomes $(12,13)$. As a result, orthopedic research has concentrated on the development of alternative strategies. Recently, the concept of ACI was introduced (14) with encouraging clinical results $(15,16)$. Further developments aiming to address the disadvantages associate with the classic first generation ACI approach gave rise to the tissue engineering based, matrix assisted chondrocyte implantation (MACI). MACI relies on the use of biomaterials seeded with chondrocytes as carriers and scaffolds for cell growth to generate new, functional, articular tissue. Over the years, a number of MACI related products have been commercialized for routine clinical application (17-20). The CaReS $₫$-system used in the current case series is composed of autologous chondrocytes seeded on three-dimensional collagen I hydrogels. Since the system's introduction in 2003 more than 1000 patients suffering from chondral defects have been treated successfully with this technology (20-22).

Most techniques aiming to repair osteochondral defects involve the transplantation of an osseous or osteochondral 
cylinder to augment the subchondral bone. Complications associated with the transplantation of such cylinders include donor site morbidity in up to $30 \%$ (23), degenerative changes of the adjoining cartilage, insufficient graft integration with the surrounding cartilage (24), and graft devitalization due to deficient vascularization in $11 \%$ of cases $(25,26)$. As a result biomechanical instability and consecutive graft sintering may occur.

To avoid these technique related side effects, in the current series, the subchondral sclerosis was only partially removed to provide a sufficiently mechanically stable bed for the transplant. Moreover, to ensure the transplant's supply with nutrients, the sclerosis was spot drilled and overlaid by cancellous bone graft containing both hematopoetic and mesenchymal stem cells additionally stimulating neovascularization, bone and cartilage formation, and therefore graft integration. After a followup period of five years, patients treated with this novel combined technique for large osteochondral defect reconstruction showed excellent clinical outcomes and a significant increase in IKDC scores.

Similar results were reported by Ochs et al. after a onestep procedure involving the transplantation of cortico-cancellous bone cylinders and matrix-based ACI (Novocart ${ }^{\circledR}$ 3D, TETEC $®$ Tissue Engineering Technologies AG, Reutlingen, Germany) (27). Results showed good reconstruction of the subchondral bone plate and a significant increase in obtained activity and IKDC scores. The study included 22 patients diagnosed with osteochondritis dissecans stage III and IV. However, the average defect size was considerably smaller measuring $4,8 \mathrm{~cm}^{2}$.

Maus et al. presented 3-year-results after osseous bone grafting and consecutive matrix-based ACI performed in 13 patients with osteochondral lesions. Smaller sized, rather superficial defects ( 6 patients, average defect size 5,6 $\mathrm{cm}^{2}$ ) were treated by autologous bone grafting and a CaReS $\AA$ transplant (22). Larger sized defects were either reconstructed by transplantation of cortico-cancellous bone cylinders or by combination of both techniques. An increase of $72 \%$ for the IKDC scores was reported when compared to preoperative findings but again defect size was considerably smaller (average $8,1 \mathrm{~cm}^{2}$ ).

In conclusion, a promising alternative treatmentstrategy for large osteochondral defect reconstruction is presented, which results in excellent clinical mid-term outcomes for affected patients. However, an increased sample size is needed to confirm the observed trends. Moreover, to increase the evidence level, the approch should be compared to other conventionally applied methods in a blinded, randomized trial.

This research did not receive any specific grant from funding agencies in the public, commercial, or not-for-profit sectors.

\section{References}

1. Attmanspacher W, Dittrich V, Stedtfeld HW. Experiences with arthroscopic therapy of chondral and osteochondral defects of the knee joint with OATS (Osteochondral Autograft Transfer System. Zentralbl Chir. 2000;125(6):494-499.

2. Alford JW, Cole BJ. Cartilage restoration, part 2: techniques, outcomes, and future directions. Am J Sports Med.2005 ;33(3):443-460; Doi: $10.1177 / 0363546505274578$

3. Outerbridge HK, Outerbridge RE, Smith DE. Osteochondral defects in the knee. A treatment using lateral patella autografts. Clin Orthop Relat Res. 2000;377:145-151.

4. Hangody L, Fules P. Autologous osteochondral mosaicplasty for the treatment of full-thickness defects of weight-bearing joints: ten years of experimental and clinical experience. J Bone Joint Surg Am. 2003;85(A Suppl 2):25-32.

5. Fritz J, Janssen P, Gaissmaier C, Schewe B, Weise K. Articular cartilage defects in the knee--basics, therapies and results. Injury. 2008;39(Suppl 1):S50-S57. Doi: 10.1016/j.injury.2008.01.039.

6. Harris JD, Siston RA, Pan X, Flanigan DC. Autologous chondrocyte implantation: a systematic review. J Bone Joint Surg Am. 2010;92(12):2220-2233. Doi: 10.2106/JBJS.J.00049.

7. Peterson L, Brittberg M, Kiviranta I, Akerlund EL, Lindahl A. Autologous chondrocyte transplantation. Biomechanics and long-term durability. Am J Sports Med. 2002;30(1):2-12. DOI: 10.1177/03635465020300011601

8. Kon E, Verdonk P, Condello V, Delcogliano M, Dhollander A, Filardo $\mathrm{G}$, et al. Matrix-assisted autologous chondrocyte transplantation for the repair of cartilage defects of the knee: systematic clinical data review and study quality analysis. Am J Sports Med. 2009;37(Suppl 1):156S-66S. Doi: 10.1177/0363546509351649

9. Grigolo B, Lisignoli G, Piacentini A, Fiorini M, Gobbi P, Mazzotti $G$, et al. Evidence for re differentiation of human chondrocytes grown on a hyaluronan-based biomaterial (HYAff 11): molecular, immunohistochemical and ultra structural analysis. Biomaterials. 2002;23(4):1187-1195. Doi:.1016/S0142-9612(01)00236-8

10. Brittberg M, Winalski CS. Evaluation of cartilage injuries and repair. J Bone Joint Surg Am. 2003;85(A Suppl 2):58-69.

11. Behrens P, Bosch U, Bruns J, Erggelet C, Esenwein SA, Gaissmaier $\mathrm{C}$, et al. [Indications and implementation of recommendations of the working group "Tissue Regeneration and Tissue Substitutes" for autologous chondrocyte transplantation (ACT)]. Z Orthop Ihre Grenzgeb. 2004;142(5):529-539.

12. Minzlaff P, Braun S, Haller B, Wortler K, Imhoff AB. [Autologous transfer of the posterior femoral condyle for large osteochondral lesions of the knee: 5-year results of the Mega-OATS technique]. Orthopade. 2010;39(6):631-636. Doi: 10.1007/s00132-010-1608-2

13. Clar H, Pascher A, Kastner N, Gruber G, Robl T, Windhager R. Matrixassisted autologous chondrocyte implantation into a $14 \mathrm{~cm}(2)$ cartilage defect, caused by steroid-induced osteonecrosis. Knee. 2010;17(3):255-257. Doi: doi: 10.1016/j.knee.2009.09.004

14. Brittberg M, Lindahl A, Nilsson A, Ohlsson C, Isaksson O, Peterson L. Treatment of deep cartilage defects in the knee with autologous chondrocyte transplantation. N Engl J Med. 1994;331(14):889-895.

15. Browne JE, Anderson AF, Arciero R, Mandelbaum B, Moseley JB, Jr., Micheli LJ, et al. Clinical outcome of autologous chondrocyte implantation at 5 years in US subjects. Clin Orthop Relat Res. 2005;(436):237-245.

16. Farr J, Rawal A, Marberry KM. Concomitant meniscal allograft 
transplantation and autologous chondrocyte implantation: minimum 2-year follow-up. Am J Sports Med. 2007;35(9):1459-1466.

17. Selmi TA, Verdonk P, Chambat P, Dubrana F, Potel JF, Barnouin L, et al. Autologous chondrocyte implantation in a novel alginate-agarose hydrogel: outcome at two years. J Bone Joint Surg Br. 2008;90(5):597604. Doi: 10.1302/0301-620X.90B5.20360

18. Ferruzzi A, Buda R, Faldini C, Vannini F, Di Caprio F, Luciani D, et al. Autologous chondrocyte implantation in the knee joint: open compared with arthroscopic technique. Comparison at a minimum follow-up of five years. J Bone Joint Surg Am. 2008;90(Suppl 4):90101

19.Pavesio A, Abatangelo G, Borrione A, Brocchetta D, Hollander AP, Kon E, et al. Hyaluronan-based scaffolds (Hyalograft $C$ ) in the treatment of knee cartilage defects: preliminary clinical findings. Novartis Found Symp. 2003;249:203-217.

20. Andereya S, Maus U, Gavenis K, Muller-Rath R, Miltner O, Mumme T, et al. First clinical experiences with a novel 3D-collagen gel (CaReS) for the treatment of focal cartilage defects in the knee. Z Orthop Ihre Grenzgeb. 2006;144(3):272-280

21. Andereya S, Maus U, Gavenis K, Gravius S, Stanzel S, Muller-Rath R, et al. [Treatment of patellofemoral cartilage defects utilizing a 3D collagen gel: two-year clinical results]. Z Orthop Unfall. 2007;145(2):139-45. doi: $10.1055 / \mathrm{s}-2007-965181$
22. Maus U, Schneider U, Gravius S, Muller-Rath R, Mumme T, Miltner O, et al. [Clinical results after three years use of matrix-associated ACT for the treatment of osteochondral defects of the knee]. Z Orthop Unfall. 2008;146(1):31-7. doi: 10.1055/s-2007-989353

23. LaPrade RF, Botker JC. Donor-site morbidity after osteochondral autograft transfer procedures. Arthroscopy. 2004;20(7):e69-73. doi: 10.1016/j.arthro.2004.06.022

24. Rose T, Craatz S, Hepp P, Raczynski C, Weiss J, Josten C, et al. The autologous osteochondral transplantation of the knee: clinical results, radiographic findings and histological aspects. Arch Orthop Trauma Surg. 2005;125(9):628-637. doi: 10.1007/s00402-005-0010-8

25. Link TM, Mischung J, Wortler K, Burkart A, Rummeny EJ, Imhoff AB. Normal and pathological MR findings in osteochondral autografts with longitudinal follow-up. Eur Radiol. 2006;16(1):88-96. doi: 10.1007/ s00330-005-2818-6

26. Salzmann GM, Paul J, Bauer JS, Woertler K, Sauerschnig M, Landwehr S, et al. T2 assessment and clinical outcome following autologous matrix-assisted chondrocyte and osteochondral autograft transplantation. Osteoarthritis Cartilage. 2009;17(12):1576-82. doi: doi.org/10.1016/j.joca.2009.07.010

27. Ochs BG, Muller-Horvat C, Rolauffs B, Fritz J, Weise K, Schewe B. [Treatment of osteochondritis dissecans of the knee: one-step procedure with bone grafting and matrix-supported autologous chondrocyte transplantation]. Z Orthop Unfall. 2007;145(2):146-151. doi: $10.1055 / \mathrm{s}-2007-965167$ 